\title{
Shaping of the Inner Solar System by the Gas-Driven Migration of Jupiter
}

\author{
Kevin J. Walsh ${ }^{1}$, Alessando Morbidelli ${ }^{2}$, Sean N. Raymond ${ }^{3}$, \\ David P. O'Brien ${ }^{4}$ and Avi M. Mandell ${ }^{5}$ \\ ${ }^{1}$ Southwest Research Institute \\ 1050 Walnut St. Suite 400, Boulder, CO, 80302, USA \\ email: kwalsh@boulder.swri.edu \\ ${ }^{2}$ Obs. Côte d'Azur, Nice, France ${ }^{3}$ Lab. d'Astrophysique de Bordeaux, Floirac France \\ ${ }^{4}$ Planetary Science Institute, Tucson, AZ, USA ${ }^{5}$ NASA Goddard, Greenbelt, MD, USA
}

\begin{abstract}
A persistent difficulty in terrestrial planet formation models is creating Mars analogs with the appropriate mass: Mars is typically an order of magnitude too large in simulations. Some recent work found that a small Mars can be created if the planetesimal disk from which the planets form has an outermost edge at 1.0 AU. However, that work and no previous work could produce a truncation of the planetesimal disk while also explaining the mass and structure of the asteroid belt. We show that gas-driven migration of Jupiter inward to $1.5 \mathrm{AU}$, before its subsequent outward migration, can truncate the disk and repopulate the asteroid belt. This dramatic migration history of Jupiter suggests that the dynamical behavior of our giant planets was more similar to that inferred for extra-solar planets than previously thought, as both have been characterised by substantial radial migration.
\end{abstract}

Keywords. Planet Formation, Planet Migration, Asteroid Belt

\section{Introduction}

Many recent studies of planets and planetary systems around other stars have revealed that giant planets can migrate in dramatic fashion (Armitage 2007, Kley and Nelson 2012). Likewise, studies of our own Solar System find evidence for planet migration with some finding that our giant planets underwent a violent phase of close encounters and scattering, possibly ejecting a 5th giant planet (Nesvorný 2011, Nesvorný \& Morbidelli 2012, Batygin et al. 2012). While this phase of planet migration is constrained by the orbits of the giant planets and the remains of the Kuiper Belt, much earlier migration of planets in the gaseous solar nebula is more difficult to constrain, but may have been important for the formation of the terrestrial planets.

The chronology of the Solar System is anchored with the oldest solids, calcium aluminum rich inclusions (CAIs), which formed $\approx 4.568$ Gyr ago (Amelin et al. 2002; Bouvier and Wadhwa 2010). Meanwhile the gaseous solar nebula (the solar "gas-disk"), which is expected to play an important role in planetesimal and planet formation, survived only 4-5 Myr after the first solids formed (Kita et al. 2005, see also Haisch et al. 2001 for lifetimes of disks around other stars). The time of gas-disk dissipation also marks an upper limit for giant planet formation, as they need to capture their gaseous envelopes from the solar nebula before it is gone.

Meanwhile, terrestrial planets take nearly an order of magnitude longer to build than the giant planets. The measured accretion timescales for the terrestrial planets are estimated at 30-100 Myr (Kleine et al. 2009), with Mars potentially being much younger than Earth. It was recently estimated to have an accretion timescale closer to $2 \mathrm{Myr}$ 
(Dauphas \& Poormand 2011). Also, the moon-forming impact, whereby a planetary embryo impacted the Earth forming its Moon, is thought to have happened around $\sim 50 \mathrm{Myr}$ (Kleine et al. 2009).

The final re-shaping of the Solar System has been temporally linked to a "late" bombardment of the inner Solar System some 4.1-3.9 Gyr ago (Tera et al. 1974, Chapman et al. 2007, Bottke et al. 2012). The cause is thought to be an instability between the orbits of the giant planets, causing them to rapidly change orbits and scatter throughout the Solar System. This in turn destabilized many small body populations, liberating many that account for the increase in the bombardment rate on the terrestrial planets during this epoch (see the "Nice Model"; Gomes et al. 2005, Morbidelli et al. 2005, Tsiganis et al. 2005, Levison et al. 2011). This is thought to be the last major re-structuring of the Solar System.

Here, we will focus on the epoch where giant planets are fully formed, but the terrestrial planets are still accreting. This era allows for giant planets to affect the conditions for formation of the terrestrial planets, potentially playing a critical role.

\section{Overview}

A nagging problem for terrestrial planet formation models is the so-called "small Mars problem". The persistent issue found in numerical models was a much larger Mars/Earth mass ratio, 5-10× too large, than observed for the actual planets (see Raymond et al. 2009 or Morbidelli et al. 2011). This factor of 5-10 in the mass of Mars was found for different initial planetesimal surface density profiles, the distribution of mass between embryos and planetesimals, and for most giant planet orbital configurations. The hints of a solution were found in very early work that modeled subsets of the full planetesial disk for specific experiments (Wetherill 1978). More recently some simulations succeeded, but used orbits of giant planets that are inconsistent with those found today or that have ever expected to have existed in the past (Raymond et al. 2009). Both approaches found success because the disk of material from which the planets form was trucated before the planets accreted, which allowed the Mars-analogs to be isolated and then avoid growing too massive.

Recent work by Hansen (2009) took a direct approach for exploring alternate surface density profiles of the planetesimal disk, and simply started with solid material between 0.7-1.0 AU. This work found that while the Earth and Venus analogs grew to be of appropriate masses within the annulus, typically the Mars analog was scattered out of the annulus very early, and then avoided further significant accretion. The work found that the Mars analog accreted very rapidly, typically reaching full mass in under $10 \mathrm{Myr}$, while the other terrestrial planets were formed in under $30 \mathrm{Myr}$. These timescales are more rapid than what was found in most previous work using a full planetesimal disk (Chambers et al. 2001, O'Brien et al. 2006, Raymond et al. 2009). Despite the successes of formation of the planet from an annulus, there was no obvious mechanism to truncate the disk.

A possible mechanism to create a truncated disk was put forth by Walsh et al. 2011. This work relied on hydrodynamic models of giant planet migration, especially the migration of a Jupiter-mass planet due to the viscous evolution of the gas disk (Lin and Papaloizou 1986, Ward 1997, see Kley and Nelson 2012). Significant inward migration by this mechanism had previously been considered impossible in our Solar System due to the existence of the asteroid belt between $\sim 2-4 \mathrm{AU}$, and also the need for Jupiter to eventually reach its present orbital distance of 5.2 AU. The solution to getting Jupiter to reverse its migration and return to the outer Solar System came from hydrodynamic 
studies that included a pair of planets (Masset and Snellgrove 2001, Morbidelli \& Crida 2007; Pierens \& Nelson 2008, Pierens and Raymond 2011). These works found that the convergent migration of the outer planet (Saturn-mass) towards the inner planet (Jupiter-mass) resulted in their capture in the 2:3 mean motion resonance (MMR). In this close configuration the gaps they cut in the gas-disk partially overlap, resulting in the negation of the inward type-II migration, and can result in outward migration for the Jupiter/Saturn mass ratio.

The precise evolution of Jupiter and Saturn in the gas disk depends on many unknown properties of the disk as well as the uncertain growth rates and formation locations for giant planets. Therefore, a priori one cannot prescribe an evolution for the giant planets. Rather, we rely on the evidence presented in the Solar System to construct a plausible scenario. The planet formation work by Hansen (2009) found that an outer edge to the planetesimal disk at 1 AU resulted in successful models of terrestrial planet formation. Simple tests found that if Jupiter reversed its migration direction ("tacked") at $1.5 \mathrm{AU}$ this was satisfied. Therefore we use this as an anchor for this study - a "tack" for Jupiter at $1.5 \mathrm{AU}$.

However, an important consideration, and strict constraint, is the existence and properties of the asteroid belt. In the scenario described above, Jupiter migrates inward to 1.5 AU and then reverses its migration, and migrates back out beyond $5 \mathrm{AU}$. This requires that it cross the asteroid belt twice, with presumably devastating consequences. The asteroid belt contains a large number of bodies which have a taxonomic dichotomy whereby the inner regions $(a<2.8 \mathrm{AU})$ are dominated by typically volatile-poor S-class asteroids and the outer region $(a>2.8 \mathrm{AU})$ is dominated by more primitive and volatilerich C-class asteroids (Gradie \& Tedesco 1982). Beyond the taxonomic distributions, there is also a complicated orbital distribution, with eccentricities between $0-0.3$, and inclinations between $0-30^{\circ}$.

Given the uncertainties in the giant planet growth and migration, the main effort of Walsh et al. (2011) was determining if there were viable scenarios for which the giant planets could both truncate the inner disk at $1 \mathrm{AU}$ and also leave behind an asteroid belt that satisfies the mass, taxonomic and orbital requirements.

\section{Results}

The focus of the numerical tests was to test a range of reasonable values for important variables of the scenario (migration timescales, presence of Uranus and Neptune, starting location of Jupiter, mass growth of Saturn), and determine if the resulting population of planetesimals were reasonable matches for today's asteroid belt. As the actual formation location of Jupiter is unknown, it was tested at a range of values from 2.5-4.5 AU, but nominally set at $3.5 \mathrm{AU}$ due to estimates of the snow line location that is presumed to be a favorable location to form giant planets (Ciesla \& Cuzzi 2006; Lecar et al. 2006; Garaud \& Lin 2007). Any populations of small bodies inside, and some directly outside, the formation location of Jupiter will then be subject to its gravitational influence during its migration inward and then outward.

The simulations start with two separate populations of asteroids (Fig. 1). Inside the orbits of the giant planets there is a planetesimal disk extending from 0.7 to $3.0 \mathrm{AU}$. Between and beyond the giant planets is a second population of asteroids, which is expected to be more primitive and volatile-rich. We apply very simple monikers to these two large groupings of asteroids, the S-class and C-class respectively, though there would be significant diversity within each class (Burbine et al. 2002, Bus et al. 2002, DeMeo et al. 2009). 


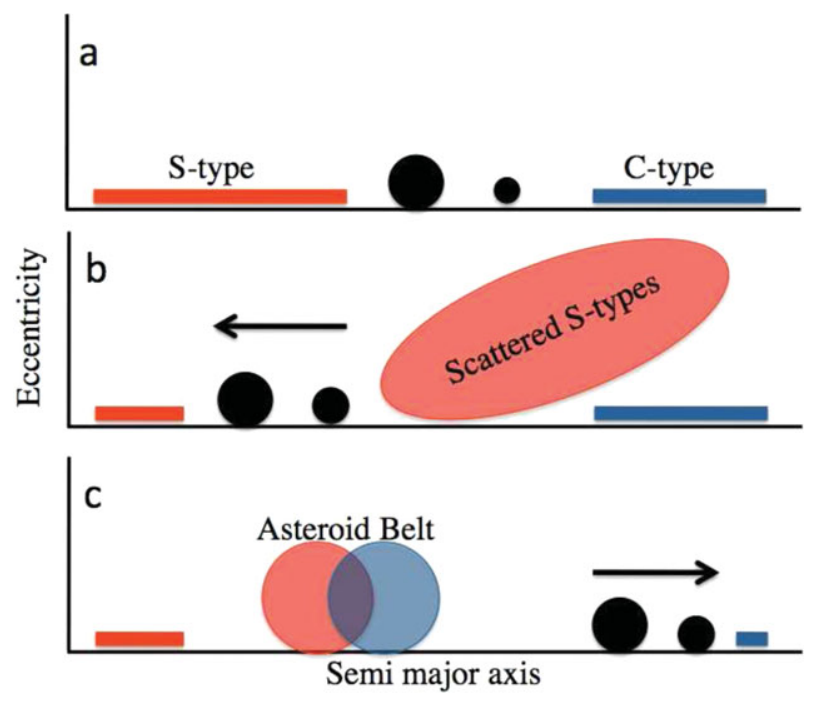

Figure 1. A sketch of the "Grand Tack" scenario. Panel (a) shows the initial conditions, with a disk of planetesimals dubbed "S-type" interior to the giant planets and "C-type" planetesimals between and beyond the giant planets. (b) Jupiter migrates inward scattering many S-type bodies and truncating the disk. (c) Jupiter migrates outward, scatter both S-type and C-type bodies back into the asteroid belt region, and the terrestrial planets eventually form from the truncated inner disk of planetesimals.

As Jupiter migrates inward it scatters about $15 \%$ of the planetesimals that it encounters, with most ending on high-eccentricity orbits beyond Jupiter. When Jupiter and Saturn begin their outward migration, they first encounter this population of scattered S-type material. Toward the end of the outward migration Jupiter and Saturn begin encountering the C-type bodies that were initially located between and beyond the giant planets. A small fraction of these asteroids are scattered inward and reach stable orbits in the asteroid belt (see Figure 2 in Walsh et al. 2011).

Asteroid Belt The final population of planetesimals in the asteroid belt region is a mixture of material from the two parent populations. The S-type material is preferentially located in the inner regions (interior to $2.8 \mathrm{AU}$ ) and the C-type material in the outer regions. This is due to the relative timing of the encounters, where the outward migrating giant planets first encounter the previously scattered S-types, and later encounter the still untouched C-types.

This matches the broad taxonomic distributions observed, with S-class bodies predominate in the inner belt and C-class in the outer belt (Gradie and Tedesco 1982). Two major classes of meteorites, ordinary and carbonaceous chondrites, are thought to originate from these S- and C-classes of asteroids respectively, and they are substantially different chemically, petrographically and isotopically. Substantial physical differences such as these are not easily explained via a cooling nebula (Warren 2011) and origin at different locations in the disk is therefore a potential solution. Therefore, scattering the asteroid belt in place from two entirely separate parent populations is a reasonable proposition.

The orbital inclinations of the planetesimals ending in the asteroid belt are a very strong constraint, as they are quite resistant to later evolution. The simulation results produce a range of values that is very similar to what is found today, between $\sim 0-30^{\circ}$. Finally, the orbital eccentricities of the planetesimals ending in the asteroid belt are 

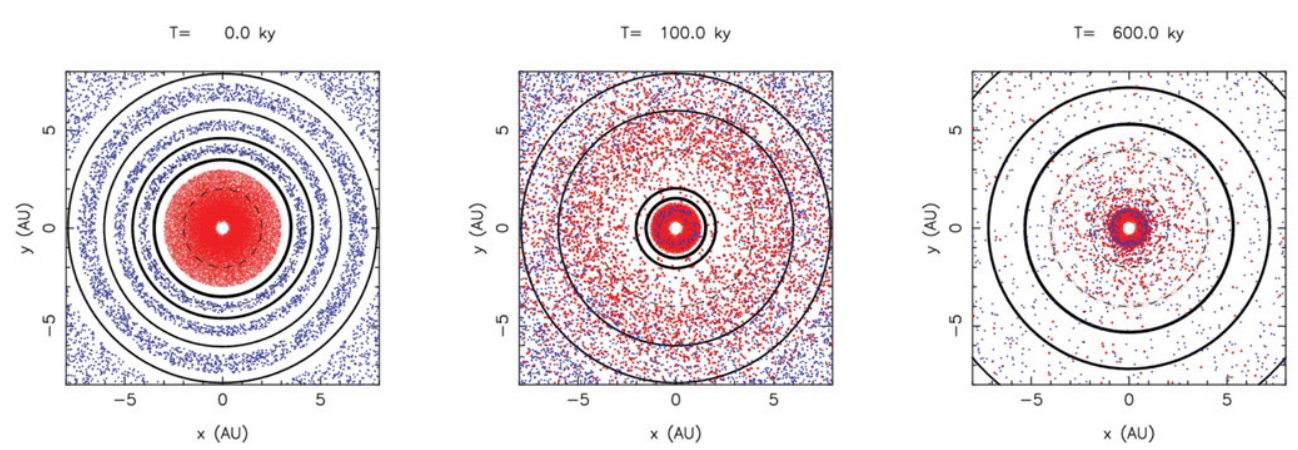

Figure 2. Three frames of the X vs. Y (AU) view of the "Grand Tack". The first shows the initial conditions, where the black circles represent the orbits of the giant planets, the red dots are the inner-disk planetesimals, and the blue dots are the outer-disk planetesimals. Moving forward in time from left to right, we track the migration of Jupiter inward to 1.5 AU, the truncation of the inner disk of planetesimals, and finally the scattering of both inner- and out-disk planetesimals into the asteroid belt region (dotted circles mark the asteroid belt) and planet-forming region.

slightly elevated when compared with today's observed asteroid belt. However, a later episode of giant planet migration has been shown to produce a reshuffling of asteroid orbital eccentricities, and thus the distribution produced in the Grand Tack will be altered at a later time (Minton and Malhotra 2009, Morbidelli et al. 2010).

Terrestrial Planets. During the inward migration of Jupiter, a susbtantial amount of the planetesimals are pushed inwards by Jupiter. After Jupiter reaches 1.5 AU, and tacks, the inner annulus has increased in mass to nearly $2.0 M_{\text {earth }}$ of material. Some of the material has significant dynamical excitement (elevated $e$ and $i$ ) due to their interactions with Jupiter - this is strongest near 1.0 AU. Otherwise, the annulus is very similar to the initial conditions used by Hansen (2009), with an edge at 1.0 AU.

The evolution of the annulus is similar to that observed by Hansen (2009), despite differences in the two models. Whereas Hansen (2009) used only embryos, Walsh et al. (2011) had mass evenly partitioned between embryos and planetesimals. The simulations presented in Walsh et al. (2011) included 8 different terrestrial planet simulations that modeled the evolution of the annulus after the migration of Jupiter, with a total of 4 simulations for each of the two different sets of initial conditions. The trend of planet mass as a function of semimajor axis was similar for both types of initial conditions and to the results of Hansen (2009). The essential trait was the trend to produce planets near a Mars mass near 1.5 AU (see Fig. 4 in Walsh et al. 2011).

There are a few classical metrics to measure the similarity of terrestrial planet simulations to the observed Solar System. First the Angular Momentum Deficit (AMD) is used to quantify the dynamical excitement of the system (see Raymond et al. 2009). The AMD for the actual planets, averaged over $10 \mathrm{Myr}$ is 0.0018 , and the simulations produced 0.0011 on average. Second, the radial mass concentration (RMC) measures how the mass is distributed between the planets (Chambers et al. 2001). The average value for the simulations is 83.8 , a close match to the current system's value of 89.9 , largely due to the close Earth/Mars mass ratios.

\section{Implications for compositions of forming planets}

There are some distinct implications for the terrestrial planets when formed from an annulus, rather than a full disk. First, the reason for the low Earth/Mars mass ratio is 
due to the isolation of the Mars-analogs after being scattered out of the annulus. By isolating the Mars-analog, it is kept from further collisions with embryos, resulting in a young accretion age. Hansen (2009) reported accretion timescales under 10 Myr for Mars-analogs, which was consistent with timescales found by Walsh et al. (2011). The cosmochemical evidence supports rapid accretion for Mars, as Nimmo \& Kleine suggest a range from 1 to $10 \mathrm{Myr}$, while Dauphas \& Pourmand (2011) findings with a Hf-W-Th chronometer point to a half-accretion timescale of 2 Myr.

One fundamental aspect of the Grand Tack model is the inclusion of primitive planetesimals that are scattered into the asteroid belt region. This is a necessary piece of the scenario needed to satisfy the constraints of the asteroid belt. This also results in primitive planetesimals being scattered into the planet-forming annulus, or being scattered onto orbits that cross the planet-forming region. The total mass of primitive planetesimals that reach planet-crossing orbits is determined by the total mass that is implanted in the asteroid belt, and is not a free variable. The total mass accreted by the Earthanalogs is typically $1-3 \%$ of the total planet mass. If the primitive planetesimals are $10 \%$ water by mass, the Earth-analog accretes about $10^{-3}$ Earth masses of water. This is largely in line with the best estimates for the total water content of the Earth (Lecuyer, M., Gillet, P. \& Robert, F. 1998; Marty 2012). This total accretion of primitive planetesimals is also consistent with geochemical constraints for total accretion of carbonaceous chondrite material to a dry proto-Earth (Marty 2012).

\section{Conclusion}

At the end of the Grand Tack, the giant planets have migrated to the outer Solar System and the asteroid belt has been scattered into place, while the terrestrial planets will still require 10's of Myr to finish accreting. Hydrodynamic simulations suggest that when the gaseous disk dissipates the giant planets will be locked in a resonant configuration (Morbidelli et al. 2007). This close and compact orbital configuration is not how we find the giant planets today, and while many of the possible resonant configurations appear to be dynamically stable for billion year timescales (Batygin and Brown 2010, Levison et al. 2011), their evolution to today's orbits left significant marks across the Solar System. The most popular flavor of this later migration/instability of the giant planets has been dubbed the "Nice Model" (Gomes et al. 2005, Tsiganis et al. 2005, Morbidelli et al. 2005, Levison et al. 2011).

These later events are important as they mark the last major structural alterations to the Solar System. The instability between the giant planets, and their subsequent migration, is driven by the massive disk beyond the giant planets - the precursor to today's Kuiper Belt. The current structure of this Kuiper Belt tells a story about the evolution of the giant planets, as do other small body populations such as the Trojans at Jupiter and the Irregular satellites of the giant planets (Morbidelli et al. 2005, Nesvorný et al. 2007). An important aspect of this giant planet migration is that as the giant planets migrate, resonances sweep through the asteroid belt, affecting the orbital distributions and depleting some of the mass. The current structure of the asteroid belt has helped to constrain the nature and timing of this late migration of the giant planets.

Thus these two epochs of giant planet migration, the Grand Tack happening early while in the presence of the gas-disk, and the Nice Model happening later, are intimately linked in a number of ways. Together they tell a dramatic story of how giant planet migration shaped our Solar System - which makes our Solar System much like those that we are finding around other stars! 


\section{Acknowledgments}

KJW acknowledges funding on this project from the Helmholtz Alliance and NLSI CLOE.

\section{References}

Amelin, Y., Krot, A. N., Hutcheon, I. D., \& Ulyanov, A. A. 2002, Science, 297, 1678

Armitage, P. J. 2007, The Astrophysical Journal, 665, 1381

Batygin, K., Brown, M. E., \& Betts, H. 2012, The Astrophysical Journal, 744, L3

Bottke, W. F., Vokrouhlický, D., Minton, D., Nesvorný, D., Morbidelli, A., Brasser, R., Simonson, B., \& Levison, H. F. 2012, Nature, 485, 78

Bouvier, A. \& Wadhwa, M. 2010, Nature Geoscience, 3, 637

Burbine, T. H., McCoy, T. J., Meibom, A., Gladman, B., \& Keil, K. 2002, Asteroids III, 653

Bus, S. J. \& Binzel, R. P. 2002, Icarus, 158, 146

Chapman, C. R., Cohen, B. A., \& Grinspoon, D. H. 2007, Icarus, 189, 233

Chambers, J. E. 2001, Icarus, 152, 205

Ciesla, F. J. \& Cuzzi, J. N. 2006, Icarus, 181, 178

Dauphas, N. \& Pourmand, A. 2011, Nature, 473, 489

DeMeo, F. E., Binzel, R. P., Slivan, S. M., \& Bus, S. J. 2009, Icarus, 202, 160

Garaud, P. \& Lin, D. N. C. 2007, The Astrophysical Journal, 654, 606

Gomes, R., Levison, H. F., Tsiganis, K., \& Morbidelli, A. 2005, Nature, 435, 466

Gradie, J. \& Tedesco, E. 1982, Science, 216, 1405

Hansen, B. M. S. 2009, The Astrophysical Journal, 703, 1131

Kita, N. T., Huss, G. R., Tachibana, S., Amelin, Y., Nyquist, L. E., \& Hutcheon, I. D. 2005, Chondrites and the Protoplanetary Disk, 341, 558

Kley, W. \& Nelson, R. P. 2012, Annual Review of Astronomy and Astrophysics, 50, 211

Kleine, T., et al. 2009, Geochimica et Cosmochimica Acta, 73, 5150

Lecar, M., Podolak, M., Sasselov, D., \& Chiang, E. 2006, The Astrophysical Journal, 640, 1115

Levison, H. F., Morbidelli, A., Tsiganis, K., Nesvorný, D., \& Gomes, R. 2011, The Astronomical Journal, 142, 152

Lin, D. N. C. \& Papaloizou, J. 1986, The Astrophysical Journal, 309, 846

Lécuyer C., Gillet P., \& Robert F. 1998, The hydrogen isotope composition of seawater and the global water cycle, 145, 249

Marty, B. 2012, Earth and Planetary Science Letters, 313, 56

Masset, F. \& Snellgrove, M. 2001, Monthly Notices of the Royal Astronomical Society, 320, L55

Minton, D. A. \& Malhotra, R. 2009, Nature, 457, 1109

Morbidelli, A., Levison, H. F., Tsiganis, K., \& Gomes, R. 2005, Nature, 435, 462

Morbidelli, A. \& Crida, A. 2007, Icarus, 191, 158

Morbidelli, A., Tsiganis, K., Crida, A., Levison, H. F., \& Gomes, R. 2007, The Astronomical Journal, 134, 1790

Morbidelli, A., Brasser, R., Gomes, R., Levison, H. F., \& Tsiganis, K. 2010, The Astronomical Journal, 140, 1391

Nesvorný, D., Vokrouhlický, D., \& Morbidelli, A. 2007, The Astronomical Journal, 133, 1962

Nesvorný, D. 2011, The Astrophysical Journal 742 L22

Nesvorný, D. \& Morbidelli, A. 2012, The Astronomical Journal, 144, 117

Nimmo, F. \& Kleine, T. 2007, Icarus, 191, 497

O'Brien, D. P., Morbidelli, A., \& Levison, H. F. 2006, Icarus, 184, 39

Pierens, A. \& Nelson, R. P. 2008, Astronomy and Astrophysics, 482, 333

Pierens, A. \& Raymond, S. N. 2011, Astronomy and Astrophysics, 533, A131

Raymond, S. N., O'Brien, D. P., Morbidelli, A., \& Kaib, N. A. 2009, Icarus, 203, 644

Tera, F., Papanastassiou, D. A., \& Wasserburg, G. J. 1974, Earth and Planetary Science Letters, 22,1

Tsiganis, K., Gomes, R., Morbidelli, A., \& Levison, H. F. 2005, Nature 435, 459 
Walsh, K. J., Morbidelli, A., Raymond, S. N., O'Brien, D. P., \& Mandell, A. M. 2011, Nature, 475,206

Ward, W. R. 1997, Icarus, 126, 261

Warren, P. H. 2011, LPI Contributions, 1639, 9027

Wetherill, G. W. 1978, IAU Colloq. 52: Protostars and Planets, 565 